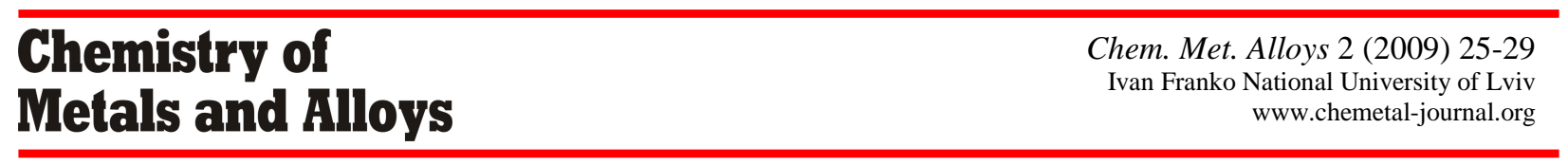

\title{
Crystal structure of the complex $\mathrm{K}_{4}\left[\mathrm{~W}_{2}(\mathrm{CN})_{10} \mathrm{O}_{3}\right] \cdot 4 \mathrm{H}_{2} \mathrm{O}$
}

\author{
Iryna TYPILO ${ }^{1}$, Olha SEREDA ${ }^{2}$, Helen STOECKLI-EVANS ${ }^{2}$, Dariya SEMENYSHYN ${ }^{1}$, Roman \\ GLADYSHEVSKII ${ }^{3}$ \\ ${ }^{1}$ Institute of Chemistry and Chemical Engineering, National University "Lvivska Polytechnika”, \\ Bandera St. 12, UA-79013 Lviv, Ukraine \\ ${ }^{2}$ Institut de Microtechnique, Université de Neuchâtel, \\ Rue Emile-Argand 11, C.P. 158, CH-2009 Neuchâtel, Switzerland \\ ${ }^{3}$ Department of Inorganic Chemistry, Ivan Franko National University of Lviv, \\ Kyryla i Mefodiya St. 6, UA-79005 Lviv, Ukraine \\ * Corresponding author.Tel.: +380-32-2582768; e-mail: semenyshyn@polynet.lviv.ua
}

Received January 26, 2009; accepted June 30; available on-line November 16, 2009

The complex $\mathrm{K}_{4}\left[\mathrm{~W}_{2}(\mathrm{CN})_{10} \mathrm{O}_{3}\right] \cdot 4 \mathrm{H}_{2} \mathrm{O}$ was prepared by hydrolysis of $\mathrm{K}_{4}\left[\mathrm{~W}(\mathrm{CN})_{8}\right] \cdot 2 \mathrm{H}_{2} \mathrm{O}$ and its crystal structure solved by direct methods. Crystal data: $\mathrm{C}_{10} \mathrm{H}_{8} \mathrm{~K}_{4} \mathrm{~N}_{10} \mathrm{O}_{7} \mathrm{~W}_{2}, M_{\mathrm{r}}=904.34$, triclinic system, space group $P \overline{1}$, cell parameters $a=8.7910(8), b=8.8424(8), c=9.4743(9) \AA, \alpha=82.316(11), \beta=64.843(10), \gamma=64.945(10)^{\circ}$, $V=602.92(10) \AA^{3}, Z=1, D_{\mathrm{X}}=2.491 \mathrm{~g} \mathrm{~cm}^{-3}, R=0.0236$ and $w R=0.0584$ for 2175 independent reflections. The coordination polyhedra of the tungsten(VI) atoms are pentagonal bipyramids $\mathrm{W}(\mathrm{CN})_{5} \mathrm{O}_{2}$, which are linked into pairs by sharing one of the oxygen atoms and twisted by $36^{\circ}$ with respect to each other. The hydrogen bonds interconnect the pairs of pentagonal bipyramids into infinite complex chains. The coordination polyhedra of the two independent potassium atoms are trigonal prisms with one capped face (by a NC unit), but have different composition, $\mathrm{K}(1)(\mathrm{NC})_{4} \mathrm{O}\left(\mathrm{OH}_{2}\right)_{2}$ and $\mathrm{K}(2)(\mathrm{NC})_{5}\left(\mathrm{OH}_{2}\right)_{2}$. The formula of this coordination compound can be written as $\mathrm{K}_{4}\left[\mathrm{OW}(\mathrm{CN})_{5} \mathrm{OW}(\mathrm{CN})_{5} \mathrm{O}\right] \cdot 4 \mathrm{H}_{2} \mathrm{O}$.

Tungsten(VI) complex / Crystal structure / Coordination cyanide

\section{Introduction}

The last years there has been increasing interest in the synthesis and crystal structure determinations of complexes containing $\left[M(\mathrm{CN})_{8}\right]^{3-/ 4-}$ anions $(M=$ Mo, $\mathrm{W})$ because of their interesting magnetic properties [14]. There exist only a few reports on investigations of cyanide complexes of tungsten and molybdenum that contain oxygen atoms. The synthesis and IR spectroscopy studies of molybdenum(IV) complexes $\mathrm{K}_{4}\left[\mathrm{MoO}_{2}(\mathrm{CN})_{4}\right] \cdot 6 \mathrm{H}_{2} \mathrm{O}$, $\mathrm{K}_{4}\left[\mathrm{MoO}_{2}(\mathrm{CN})_{4}\right]$ $\mathrm{K}_{3}\left[\mathrm{MoO}(\mathrm{OH})(\mathrm{CN})_{4}\right], \quad \mathrm{K}_{2}\left[\mathrm{Mo}(\mathrm{OH})_{2}(\mathrm{CN})_{4}\right]$, $\mathrm{Cd}\left(\mathrm{H}_{2} \mathrm{O}\right)_{6}\left[\mathrm{Mo}(\mathrm{OH})_{2}(\mathrm{CN})_{4}\right], \quad \mathrm{Mo}(\mathrm{OH})_{2}(\mathrm{CN})_{2} \cdot \mathrm{H}_{2} \mathrm{O}$, $\mathrm{K}_{6}\left[\mathrm{Mo}^{4+}{ }_{2} \mathrm{Mo}^{6+}(\mathrm{CN})_{8} \mathrm{O}_{6}\right] \cdot 2 \mathrm{H}_{2} \mathrm{O}$ are described in [5]. The authors of [6] report on photolysis of $\mathrm{Na}_{4}\left[\mathrm{~W}(\mathrm{CN})_{8}\right]$ that led to the formation of the complex $\mathrm{Na}_{4}\left[\mathrm{WO}_{2}(\mathrm{CN})_{4}\right] \cdot 12 \mathrm{H}_{2} \mathrm{O}$. The crystal structure of the complex $\mathrm{Na}_{3}\left[\mathrm{MoO}(\mathrm{OH})(\mathrm{CN})_{4}\right] \cdot 4 \mathrm{H}_{2} \mathrm{O}$ belongs to space group $P 2_{1} / m$ [7] and crystallographic data for $\mathrm{Rb}_{3}\left[\mathrm{WO}_{2}(\mathrm{CN})_{3} \mathrm{H}_{2} \mathrm{O}\right] \cdot 3 \mathrm{H}_{2} \mathrm{O}$ are the following: monoclinic system, space group $P 2_{1}$, cell parameters $a$ $=12.047(3), b=7.980(2), c=7.815(2) \AA, \gamma=$ $108.05(2)^{\circ}[8]$. Here we present the crystal structure of a novel complex, $\mathrm{K}_{4}\left[\mathrm{~W}_{2}(\mathrm{CN})_{10} \mathrm{O}_{3}\right] \cdot 4 \mathrm{H}_{2} \mathrm{O}$.

\section{Experimental}

\section{Synthesis}

The complex $\mathrm{K}_{4}\left[\mathrm{~W}_{2}(\mathrm{CN})_{10} \mathrm{O}_{3}\right] \cdot 4 \mathrm{H}_{2} \mathrm{O}$ was prepared by hydrolysis of $\mathrm{K}_{4}\left[\mathrm{~W}(\mathrm{CN})_{8}\right] \cdot 2 \mathrm{H}_{2} \mathrm{O}$. A solution of $\mathrm{K}_{4}\left[\mathrm{~W}(\mathrm{CN})_{8}\right] \cdot 2 \mathrm{H}_{2} \mathrm{O}$ was stored in dark at room temperature. Orange, well-shaped crystals, suitable for single-crystal diffraction, were formed after several months.

\section{$X$-ray diffraction}

The single crystal of $\mathrm{K}_{4}\left[\mathrm{~W}_{2}(\mathrm{CN})_{10} \mathrm{O}_{3}\right] \cdot 4 \mathrm{H}_{2} \mathrm{O}$ selected for the diffraction experiment was approximately $0.50 \times 0.25 \times 0.20 \mathrm{~mm}$ large. Intensity data were collected at $173 \mathrm{~K}$ on a STOE IPDS device [9] using graphite-monochromated $\mathrm{Mo} \mathrm{K} \alpha$ radiation (image plate distance $70 \mathrm{~mm}, \phi$ oscillation scans $0-200^{\circ}$, step $\Delta \phi=1.0^{\circ}$, exposure time $3 \mathrm{~min}, \theta$ range $2.38-25.83^{\circ}$ ). The structure was solved by direct methods using programs from SHELX-97 [10,11]. The non-hydrogen atoms were refined anisotropically from weighted fullmatrix least-squares on $F^{2}$; hydrogen atoms were located from Fourier difference maps. An empirical absorption correction was applied using the 
Table 1 Experimental details of the structure refinement for $\mathrm{K}_{4}\left[\mathrm{~W}_{2}(\mathrm{CN})_{10} \mathrm{O}_{3}\right] \cdot 4 \mathrm{H}_{2} \mathrm{O}$.

\begin{tabular}{|c|c|}
\hline Crystal color & yellow \\
\hline Crystal size, $\mathrm{mm}$ & $0.50 \times 0.25 \times 0.20$ \\
\hline Empirical formula & $\mathrm{C}_{10} \mathrm{H}_{8} \mathrm{~K}_{4} \mathrm{~N}_{10} \mathrm{O}_{3} \mathrm{~W}_{2}$ \\
\hline$M_{\mathrm{r}}$ & 904.34 \\
\hline System & triclinic \\
\hline Space group & $P \overline{1}$ \\
\hline$a, \AA$ & $8.7910(8)$ \\
\hline$b, \AA$ & $8.8424(8)$ \\
\hline$c, \AA$ & $9.4743(9)$ \\
\hline$\alpha,{ }^{\circ}$ & $82.316(11)$ \\
\hline$\beta,{ }^{\circ}$ & $64.843(10)$ \\
\hline$\gamma,^{o}$ & $64.945(10)$ \\
\hline$V, \AA^{3}$ & $602.92(10)$ \\
\hline$Z$ & 1 \\
\hline$D_{\mathrm{X}}, \mathrm{g} \mathrm{cm}^{-3}$ & 2.491 \\
\hline$\mu, \mathrm{mm}^{-1}$ & 10.274 \\
\hline Radiation & Mo $\mathrm{K} \alpha$ \\
\hline$\lambda, \AA$ & 0.71073 \\
\hline Range $\theta,^{\circ}$ & $2.38-25.83$ \\
\hline Number of measured reflections & 4761 \\
\hline Number of independent reflections & 2175 \\
\hline Number of reflections with $I>2 \sigma(I)$ & 2069 \\
\hline$R_{\text {int }}$ & 0.0451 \\
\hline Number of refined parameters & 151 \\
\hline$R, w R, S$ & $0.0236,0.0584,1.121$ \\
\hline$R, w R(I>2 \sigma(I))$ & $0.0221,0.0580$ \\
\hline$\rho_{\min } / \rho_{\max }$, e $\AA^{-3}$ & $-1.411 / 0.948$ \\
\hline$T, \mathrm{~K}$ & $173(2)$ \\
\hline
\end{tabular}

Table 2 Atomic coordinates and equivalent/isotropic displacement parameters for $\mathrm{K}_{4}\left[\mathrm{~W}_{2}\left(\mathrm{CN}_{10} \mathrm{O}_{3}\right] \cdot 4 \mathrm{H}_{2} \mathrm{O}\right.$.

\begin{tabular}{l|l|l|l|l|l}
\hline Atom & $\begin{array}{l}\text { Wyckoff } \\
\text { position }\end{array}$ & \multicolumn{1}{|c|}{$x$} & \multicolumn{1}{|c}{$z$} & $U_{\text {eq }}, \AA^{2}$ \\
\hline $\mathrm{K}(1)$ & $2 i$ & $0.32902(16)$ & $0.41507(16)$ & $0.21352(13)$ & $0.0307(3)$ \\
$\mathrm{K}(2)$ & $2 i$ & $0.56862(17)$ & $0.26959(17)$ & $0.59955(14)$ & $0.0367(4)$ \\
$\mathrm{W}$ & $2 i$ & $0.09248(2)$ & $0.11461(2)$ & $0.08357(2)$ & $0.0158(1)$ \\
$\mathrm{C}(1)$ & $2 i$ & $0.0444(6)$ & $0.3019(6)$ & $0.9137(5)$ & $0.0220(14)$ \\
$\mathrm{C}(2)$ & $2 i$ & $0.2834(6)$ & $0.8641(6)$ & $0.1016(5)$ & $0.0191(14)$ \\
$\mathrm{C}(3)$ & $2 i$ & $0.3391(6)$ & $0.0382(6)$ & $0.8659(5)$ & $0.0230(12)$ \\
$\mathrm{C}(4)$ & $2 i$ & $0.8079(6)$ & $0.3002(6)$ & $01952(5)$ & $0.0218(14)$ \\
$\mathrm{C}(5)$ & $2 i$ & $0.9560(6)$ & $0.0209(6)$ & $0.3053(5)$ & $0.0221(14)$ \\
$\mathrm{N}(1)$ & $2 i$ & $0.0209(6)$ & $0.3914(5)$ & $0.8189(5)$ & $0.0297(12)$ \\
$\mathrm{N}(2)$ & $2 i$ & $0.1174(6)$ & $0.0304(6)$ & $0.5814(5)$ & $0.0292(14)$ \\
$\mathrm{N}(3)$ & $2 i$ & $0.3802(6)$ & $0.7279(6)$ & $0.1073(5)$ & $0.0271(12)$ \\
$\mathrm{N}(4)$ & $2 i$ & $0.5324(6)$ & $0.0031(6)$ & $0.2483(5)$ & $0.0326(14)$ \\
$\mathrm{N}(5)$ & $2 i$ & $0.6597(6)$ & $0.3942(6)$ & $0.2581(5)$ & $0.0298(12)$ \\
$\mathrm{O}(1)$ & $2 i$ & $0.1316(5)$ & $0.6394(5)$ & $0.4826(4)$ & $0.0352(12)$ \\
$\mathrm{O}(2)$ & $2 i$ & $0.1732(5)$ & $0.2134(4)$ & $0.1637(4)$ & $0.0245(11)$ \\
$\mathrm{O}(3)$ & $2 i$ & $0.3214(5)$ & $0.2633(5)$ & $0.4959(4)$ & $0.0364(12)$ \\
$\mathrm{O}(4)$ & $1 a$ & 0 & 0 & $0.0195(14)$ \\
$\mathrm{H}(1 \mathrm{~A})$ & $2 i$ & 0.0316 & 0.7255 & 0.4905 & 0.053 \\
$\mathrm{H}(1 \mathrm{~B})$ & $2 i$ & 0.1023 & 0.5711 & 0.053 \\
$\mathrm{H}(3 \mathrm{~A})$ & $2 i$ & 0.2376 & 0.5959 & 0.054 \\
$\mathrm{H}(3 \mathrm{~B})$ & $2 i$ & 0.3633 & 0.1605 & & 0.054 \\
\hline
\end{tabular}




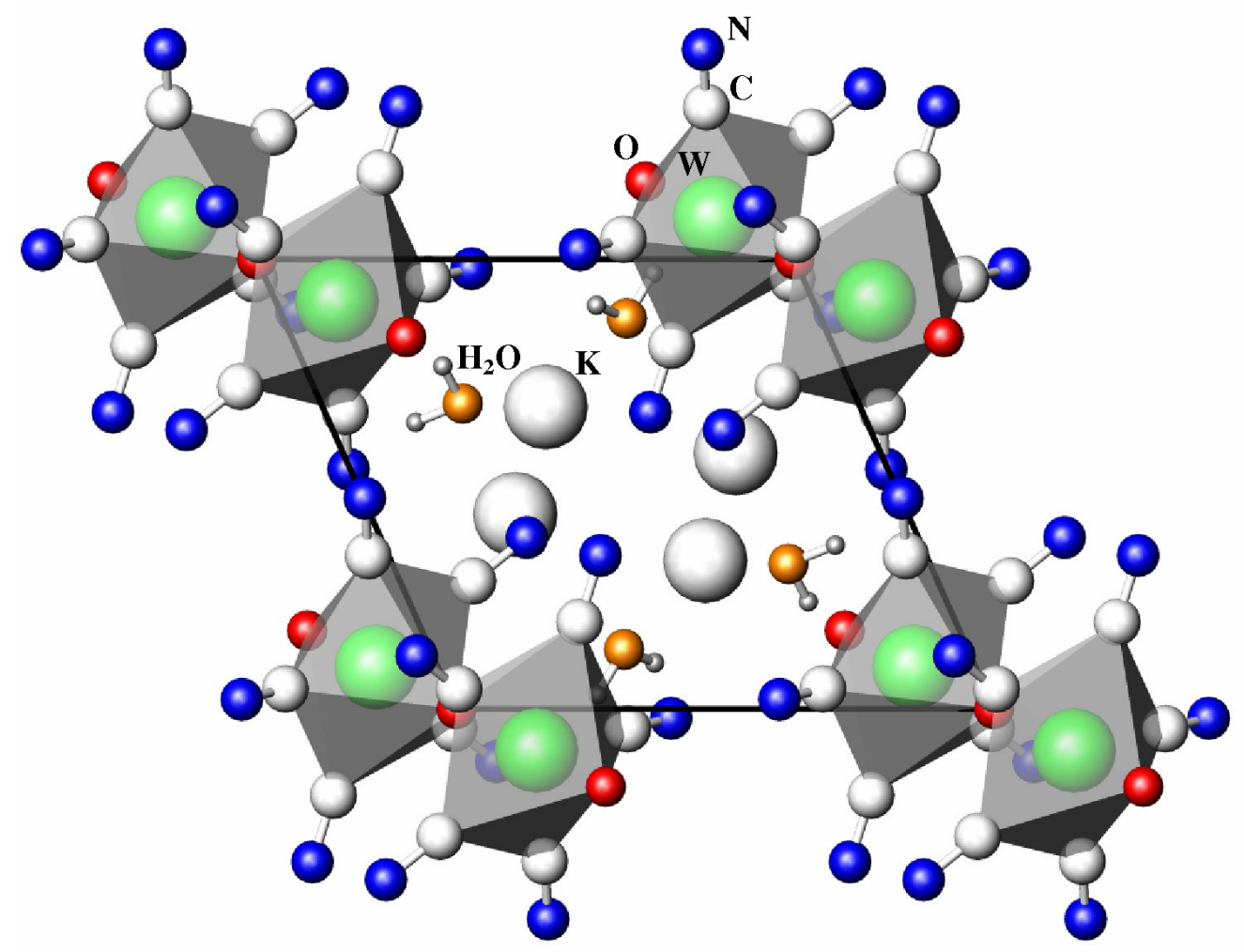

Fig. 1 Projection of the structure of $\mathrm{K}_{4}\left[\mathrm{~W}_{2}(\mathrm{CN})_{10} \mathrm{O}_{3}\right] \cdot 4 \mathrm{H}_{2} \mathrm{O}$ along [001].

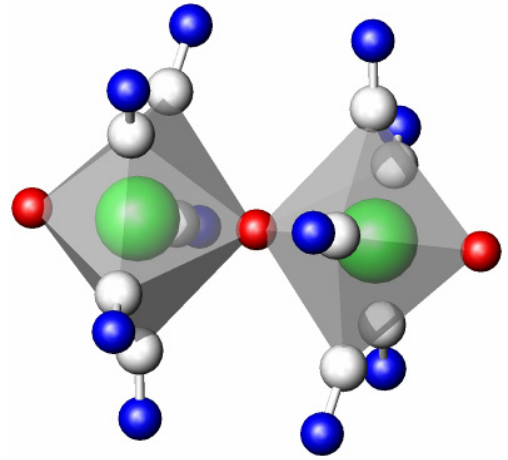

$\mathrm{W}_{2}(\mathrm{CN}){ }_{10} \mathrm{O}_{3}$

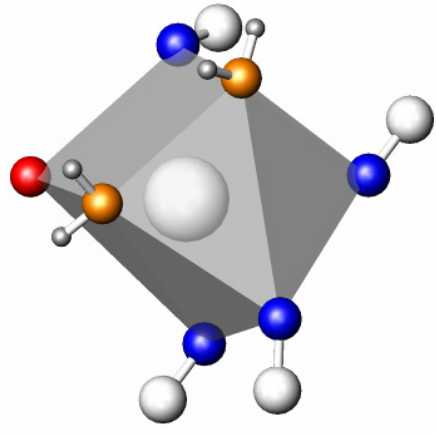

$\mathrm{K}(1)(\mathrm{NC})_{4} \mathrm{O}\left(\mathrm{OH}_{2}\right)_{2}$

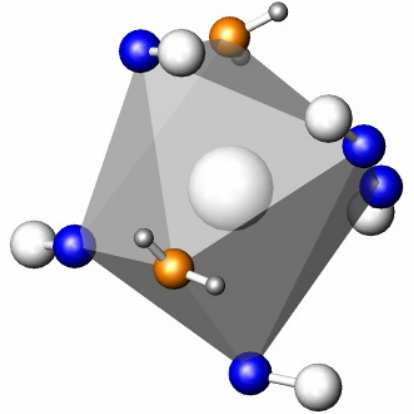

$\mathrm{K}(2)(\mathrm{NC})_{5}\left(\mathrm{OH}_{2}\right)_{2}$

Fig. 2 Coordination polyhedra of the $\mathrm{W}$ and $\mathrm{K}$ atoms in $\mathrm{K}_{4}\left[\mathrm{~W}_{2}(\mathrm{CN})_{10} \mathrm{O}_{3}\right] \cdot 4 \mathrm{H}_{2} \mathrm{O}$.

DELrefABS routine in PLATON [12]; transmission factors $T_{\min } / T_{\max }=0.086 / 0.120$. A projection of the $\mathrm{K}_{4}\left[\mathrm{~W}_{2}(\mathrm{CN})_{10} \mathrm{O}_{3}\right] \cdot 4 \mathrm{H}_{2} \mathrm{O}$ structure and the coordination polyhedra of the $\mathrm{W}$ and $\mathrm{K}$ atoms (drawn with ATOMS [13]) are presented in Figs. 1 and 2, respectively. Details of the structure investigation are given in Table 1, atomic coordinates and equivalent (isotropic for $\mathrm{H}$ atoms) displacement parameters in Table 2, and selected bond lengths and angles in Table 3.

\section{Results and discussion}

The X-ray diffraction study of the crystal structure of $\mathrm{K}_{4}\left[\mathrm{~W}_{2}(\mathrm{CN})_{10} \mathrm{O}_{3}\right] \cdot 4 \mathrm{H}_{2} \mathrm{O}$ reveals that it consists of three structural units, $\mathrm{W}_{2}(\mathrm{CN})_{10} \mathrm{O}_{3}, \mathrm{~K}(1)(\mathrm{NC})_{4} \mathrm{O}\left(\mathrm{OH}_{2}\right)_{2}$ and $\mathrm{K}(2)(\mathrm{NC})_{5}\left(\mathrm{OH}_{2}\right)_{2}$. The coordination polyhedra of the tungsten(VI) atoms are pentagonal bipyramids $\mathrm{W}(\mathrm{CN})_{5} \mathrm{O}_{2}$, which are linked into pairs by common oxygen atoms and twisted by $36^{\circ}$ with respect to each 
Table 3 Selected bond distances and angles in $\mathrm{K}_{4}\left[\mathrm{~W}_{2}(\mathrm{CN})_{10} \mathrm{O}_{3}\right] \cdot 4 \mathrm{H}_{2} \mathrm{O}$.

\begin{tabular}{|c|c|c|c|}
\hline Atoms & $\delta, \AA$ & Atoms & $\omega,^{\circ}$ \\
\hline $\mathrm{W}-\mathrm{C}(3)$ & $2.177(5)$ & $\mathrm{C}(1)-\mathrm{W}-\mathrm{C}(2)$ & $141.78(17)$ \\
\hline $\mathrm{W}-\mathrm{C}(2)$ & $2.179(5)$ & $\mathrm{C}(1)-\mathrm{W}-\mathrm{C}(3)$ & $71.63(18)$ \\
\hline W-C (5) & $2.185(5)$ & $\mathrm{C}(1)-\mathrm{W}-\mathrm{C}(4)$ & $72.69(18)$ \\
\hline $\mathrm{W}-\mathrm{C}(1)$ & $2.187(5)$ & $\mathrm{C}(1)-\mathrm{W}-\mathrm{C}(5)$ & $143.7(2)$ \\
\hline W-C(4) & $2.193(5)$ & $\mathrm{C}(2)-\mathrm{W}-\mathrm{C}(3)$ & $70.92(17)$ \\
\hline $\mathrm{W}-\mathrm{O}(2)$ & $1.733(4)$ & $\mathrm{C}(2)-\mathrm{W}-\mathrm{C}(4)$ & $143.56(18)$ \\
\hline $\mathrm{W}-\mathrm{O}(4)$ & $1.9428(3)$ & $\mathrm{C}(2)-\mathrm{W}-\mathrm{C}(5)$ & $71.79(18)$ \\
\hline $\mathrm{C}(3)-\mathrm{N}(4)$ & $1.139(7)$ & $\mathrm{C}(3)-\mathrm{W}-\mathrm{C}(4)$ & $144.19(18)$ \\
\hline $\mathrm{C}(4)-\mathrm{N}(5)$ & $1.140(7)$ & $\mathrm{C}(3)-\mathrm{W}-\mathrm{C}(5)$ & $142.32(18)$ \\
\hline $\mathrm{C}(1)-\mathrm{N}(1)$ & $1.142(6)$ & $\mathrm{C}(4)-\mathrm{W}-\mathrm{C}(5)$ & $72.03(18)$ \\
\hline $\mathrm{C}(5)-\mathrm{N}(2)$ & $1.142(7)$ & $\mathrm{O}(2)-\mathrm{W}-\mathrm{C}(1)$ & $95.69(18)$ \\
\hline $\mathrm{C}(2)-\mathrm{N}(3)$ & $1.154(7)$ & $\mathrm{O}(2)-\mathrm{W}-\mathrm{C}(2)$ & $94.11(19)$ \\
\hline $\mathrm{K}(1)-\mathrm{O}(3)$ & $2.815(4)$ & $\mathrm{O}(2)-\mathrm{W}-\mathrm{C}(3)$ & $93.8(2)$ \\
\hline $\mathrm{K}(1)-\mathrm{O}(1)$ & $2.850(4)$ & $\mathrm{O}(2)-\mathrm{W}-\mathrm{C}(4)$ & $92.55(19)$ \\
\hline $\mathrm{K}(1)-\mathrm{O}(2)$ & $2.850(5)$ & $\mathrm{O}(2)-\mathrm{W}-\mathrm{C}(5)$ & $94.02(19)$ \\
\hline $\mathrm{K}(1)-\mathrm{N}(1)$ & $2.948(6)$ & $\mathrm{O}(4)-\mathrm{W}-\mathrm{C}(1)$ & $86.06(15)$ \\
\hline $\mathrm{K}(1)-\mathrm{N}(3)$ & $2.965(5)$ & $\mathrm{O}(4)-\mathrm{W}-\mathrm{C}(2)$ & $84.92(15)$ \\
\hline $\mathrm{K}(1)-\mathrm{N}(3)$ & $3.009(5)$ & $\mathrm{O}(4)-\mathrm{W}-\mathrm{C}(3)$ & $87.41(15)$ \\
\hline $\mathrm{K}(1)-\mathrm{N}(5)$ & $3.038(6)$ & $\mathrm{O}(4)-\mathrm{W}-\mathrm{C}(4)$ & $87.29(15)$ \\
\hline $\mathrm{K}(2)-\mathrm{O}(3)$ & $2.761(5)$ & $\mathrm{O}(4)-\mathrm{W}-\mathrm{C}(5)$ & $84.15(15)$ \\
\hline $\mathrm{K}(2)-\mathrm{O}(1)$ & $2.843(5)$ & $\mathrm{O}(4)-\mathrm{W}-\mathrm{O}(2)$ & $178.12(11)$ \\
\hline $\mathrm{K}(2)-\mathrm{N}(5)$ & $2.899(5)$ & & \\
\hline $\mathrm{K}(2)-\mathrm{N}(4)$ & $2.911(5)$ & & \\
\hline$K(2)-N(2)$ & $2.930(5)$ & & \\
\hline $\mathrm{K}(2)-\mathrm{N}(3)$ & $3.001(5)$ & & \\
\hline $\mathrm{K}(2)-\mathrm{N}(5)$ & $3.148(5)$ & & \\
\hline $\mathrm{O}(1)-\mathrm{H}(1 \mathrm{~B})$ & 0.860 & & \\
\hline $\mathrm{O}(1)-\mathrm{H}(1 \mathrm{~A})$ & 0.870 & & \\
\hline $\mathrm{O}(3)-\mathrm{H}(3 \mathrm{~A})$ & 0.870 & & \\
\hline $\mathrm{O}(3)-\mathrm{H}(3 \mathrm{~B})$ & 0.890 & & \\
\hline
\end{tabular}

Table 4 Hydrogen bonds for $\mathrm{K}_{4}\left[\mathrm{~W}_{2}(\mathrm{CN})_{10} \mathrm{O}_{3}\right] \cdot 4 \mathrm{H}_{2} \mathrm{O}$.

\begin{tabular}{c|l|l|l|l}
\hline D-H...A & \multicolumn{1}{|c|}{ D-H, $\AA$ H...A, $\AA$. } & \multicolumn{1}{|c}{ D...A, $\AA$. } & \multicolumn{1}{c}{ D-H...A, $^{\circ}$} \\
\hline $\mathrm{O}(1)-\mathrm{H}(1 \mathrm{~A}) \ldots \mathrm{N}(2)$ & 0.870 & 2.190 & $2.988(7)$ & 152.0 \\
$\mathrm{O}(3)-\mathrm{H}(3 \mathrm{~A}) \ldots \mathrm{N}(1)$ & 0.870 & 2.260 & $3.024(6)$ & 146.0 \\
$\mathrm{O}(3)-\mathrm{H}(3 \mathrm{~B}) \ldots \mathrm{N}(4)$ & 0.890 & 2.130 & $2.893(6)$ & 143.0 \\
\hline
\end{tabular}

other. The $\mathrm{W}$ atom is seven-fold coordinated by five carbon atoms from $\mathrm{CN}$ groups $(\mathrm{C}(1), \mathrm{C}(2), \mathrm{C}(3), \mathrm{C}(4)$, $\mathrm{C}(5))$ and two oxygen atoms $(\mathrm{O}(2), \mathrm{O}(4))$. The W-C distances range from 2.177(5) to 2.193(5) $\AA$ and the $\mathrm{C} \equiv \mathrm{N}$ distances from $1.139(7)$ to $1.154(7) \AA$. The $\mathrm{W}$ $\mathrm{C}-\mathrm{N}$ bond angles range from $175.4(4)$ to $179.2(4)^{\circ}$ and do thus not deviate much from $180^{\circ}$.

The water molecules $(\mathrm{O}(1), \mathrm{O}(3))$ and potassium atoms are located between the $\mathrm{W}_{2}(\mathrm{CN}){ }_{10} \mathrm{O}_{3}$ units. $\mathrm{K}(1)$ is surrounded by four $\mathrm{N}$ atoms from $\mathrm{CN}$ groups $(\mathrm{N}(1)$, two $\mathrm{N}(3), \mathrm{N}(5))$, two $\mathrm{O}$ atoms from water molecules $(\mathrm{O}(1), \mathrm{O}(3))$, and one more oxygen atom $(\mathrm{O}(2)) . \mathrm{K}(2)$ is surrounded by five $\mathrm{N}$ atoms from $\mathrm{CN}$ groups $(\mathrm{N}(4)$, two $\mathrm{N}(5), \mathrm{N}(2), \mathrm{N}(3))$ and two $\mathrm{O}$ atoms from water molecules $(\mathrm{O}(1), \mathrm{O}(3))$. The coordination polyhedra of both potassium sites are trigonal prisms with one face centered by a NC group, but they have different compositions, $\mathrm{K}(1)(\mathrm{NC})_{4} \mathrm{O}\left(\mathrm{OH}_{2}\right)_{2} \quad$ and $\mathrm{K}(2)(\mathrm{NC})_{5}\left(\mathrm{OH}_{2}\right)_{2}$. The formula of the compound can conveniently be written as $\mathrm{K}_{4}\left[\mathrm{OW}(\mathrm{CN})_{5} \mathrm{OW}(\mathrm{CN})_{5} \mathrm{O}\right] \cdot 4 \mathrm{H}_{2} \mathrm{O}$.

Hydrogen bonding is observed in the structure of $\mathrm{K}_{4}\left[\mathrm{~W}_{2}(\mathrm{CN})_{10} \mathrm{O}_{3}\right] \cdot 4 \mathrm{H}_{2} \mathrm{O}$ between the water molecules $(\mathrm{H}(1 \mathrm{~A}), \mathrm{H}(3 \mathrm{~A}), \mathrm{H}(3 \mathrm{~B}))$ and $\mathrm{CN}$ groups of the $\mathrm{W}_{2}(\mathrm{CN})_{10} \mathrm{O}_{3}$ units. The hydrogen atom $\mathrm{H}(1 \mathrm{~B})$ does not form hydrogen bonding. H-bond distances in $\mathrm{K}_{4}\left[\mathrm{~W}_{2}(\mathrm{CN})_{10} \mathrm{O}_{3}\right] \cdot 4 \mathrm{H}_{2} \mathrm{O}$ are listed in Table 4. The hydrogen bonds interconnect the pairs of pentagonal bipyramids into infinite complex chains running along [10̄̄] (Fig. 3). 


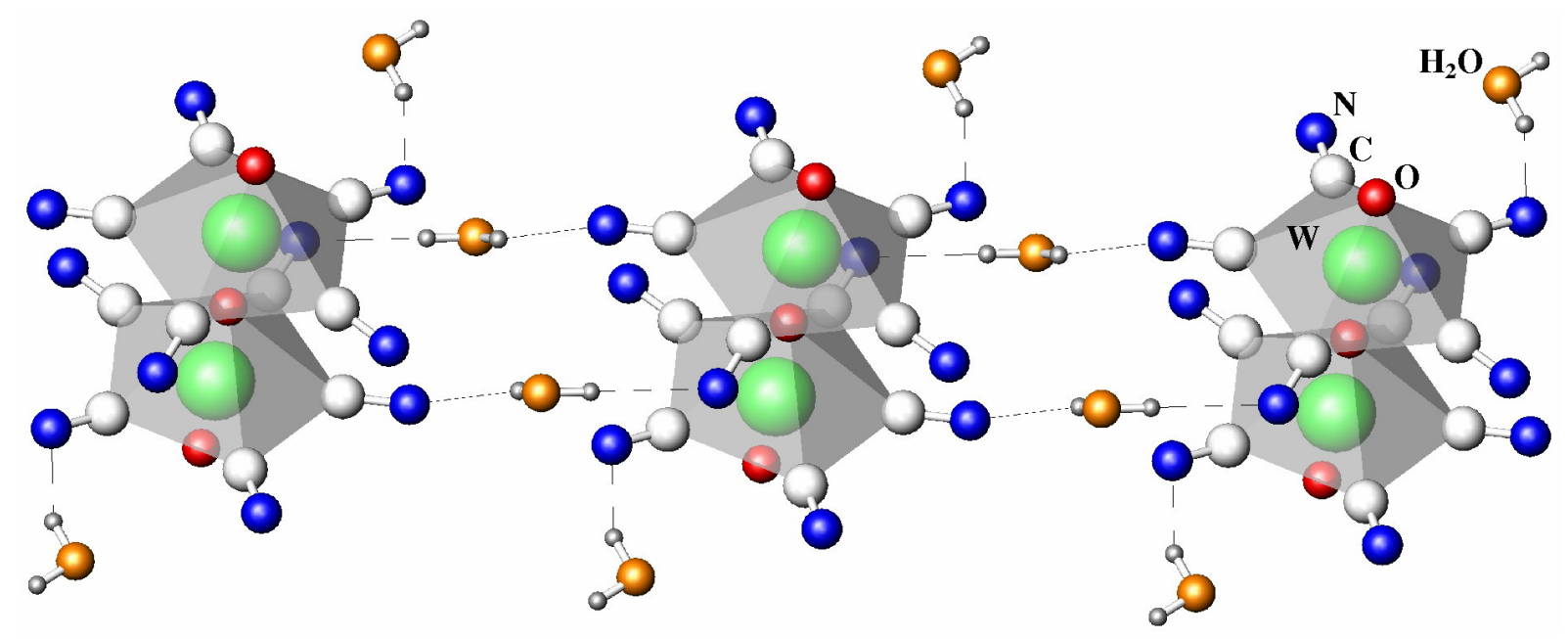

Fig. 3 Part of an infinite chain of $\mathrm{W}_{2}(\mathrm{CN})_{10} \mathrm{O}_{3}$. units interconnected via hydrogen bonding.

\section{Conclusions}

The formula of this coordination compound can be written as $\mathrm{K}_{4}\left[\mathrm{OW}(\mathrm{CN})_{5} \mathrm{OW}(\mathrm{CN})_{5} \mathrm{O}\right] \cdot 4 \mathrm{H}_{2} \mathrm{O}$. The structure is quite different from those of other tungsten-cyanide complexes and the first representative where the coordination polyhedra of the tungsten atoms form pairs.

\section{References}

[1] T. Hozumi, S. Ohkoshi, Y. Arimoto, H. Saino, Y. Mizobe, K. Hashimoto, J. Phys. Chem. B 107 (2003) 11571-11574.

[2] S. Ikeda, T. Hozumi, K. Hashimoto, S. Ohkoshi, Dalton Trans. (2005) 2120-2123.

[3] S. Kaneko, Y. Tsunobuchi, K. Nakabayashi, S. Ohkoshi, Polyhedron 28 (2009) 1893-1897.

[4] O.A. Sereda, H. Stoeckli-Evans, I.V. Typilo, D.I. Semenyshyn, R.E. Gladyshevskii, Koord. Khim. 35 (2009) 17-20.
[5] J. van de Poel, H.M. Neumann, Inorg. Chem. 7 (1968) 2086-2091.

[6] A. Samotus, M. Dudek, A. Kanas, J. Inorg. Nucl. Chem. 37(4) (1975) 943-948.

[7] K. Stadnicka, Rocz. Chem. 47(11) (1973) 20212034.

[8] B. Chernyak, R. Gladyshevskii, V. Kovbashyn, V. Yarovets, Coll. Abstr. VI All-Union Conf. Chem. Technol. Mo W, Nalchuk, 1988, p. 43.

[9] Stoe IPDS Software, Stoe \& Cie $\mathrm{GmbH}$, Darmstadt, Germany, 2000.

[10] G.M. Sheldrick, Acta Crystallogr. A 46 (1990) 467-473.

[11] G.M. Sheldrick, SHELXS-97, Universität Göttingen, Göttingen, Germany, 1999.

[12] A.L. Spek, J. Appl. Crystallogr. 36 (2003) 7-13.

[13] E. Dowty, Atoms - A Computer Program for Displaying Atomic Structures, Kingsport, TN, 1999. 